\title{
Reconstrução interna dos prefixos relacionais da língua Mawé
}

\author{
Ana Suelly Arruda Câmara Cabral ${ }^{1}$ \\ Aryon Dall'Igna Rodrigues ${ }^{2}$ \\ Dulce do Carmo Franceschini ${ }^{3}$
}

\section{Resumo}

Este artigo apresenta uma hipótese reconstrutiva dos prefixos relacionais da língua Mawé. São sublinhadas as motivações para as mudanças fonológicas sofridas nas respectivas formas desses prefixos, as quais resultaram em novos alomorfes e contribuíram para a redistribuição de temas em novas classes e subclasses.

Palavras-chave: Prefixos relacionais. Mudanças históricas. Reconstrução interna. Língua Mawé. Tronco Tupí.

\begin{abstract}
This paper deals with a reconstructive hypothesis of the Mawé relational prefixes. It highlights the linguistic motivations for the changes of their phonological forms, which have contributed to the development of new allomorphs, as well as to the redistribution of Mawé's stems into new thematic classes and subclasses.
\end{abstract}

Keywords: Relational prefixes. Historical changes. Internal reconstruction. Mawé language. Tupian stock.

\section{Introdução}

Neste artigo fundamentamos uma proposta de reconstrução interna do sistema de prefixos relacionais da língua Mawé ${ }^{4}$, único membro sobrevivente

1 Professora Associada, Instituto de Letras, Universidade de Brasília (UnB), ViceCoordenadora do Laboratório de Línguas Indígenas (LALI) e Vice-Coordenadora do Núcleo de Estudos da Amazônia do Centro de Estudos Avançados Multidisciplinares (NEAz/CEAM) da UnB. Bolsista de Produtividade Científica PQ 1D-CNPq.

2 Professor Doutor Honoris Causa, UnB e Coordenador do Laboratório de Línguas Indígenas da mesma universidade. Bolsista de Produtividade Científica PQ 1D-CNPq.

3 Professora Adjunta da Universidade Federal de Uberlândia e Pesquisadora do Laboratório de Línguas Indígenas da UnB.

4 A primeira versão desta proposta foi apresentada durante o V Congresso Internacional da ABRALIN, Belo Horizonte, MG (2007a), e durante o II Encontro sobre Línguas e Culturas Tupí, Universidade de Brasília (2007b), tendo sido posteriormente enriquecida com a colaboração de Aryon Dall'Igna Rodrigues e apresentada durante o I Encontro Internacional de Arqueologia e Linguística Histórica das Línguas Indígenas SulAmericanas, Universidade de Brasília, 2011. 
da família linguística conhecida pelo mesmo nome, a qual constitui uma das 10 famílias do tronco Tupí (cf. Rodrigues 1985a). A expressão prefixos relacionaisfoi cunhada por Rodrigues para nomear um conjunto de prefixos que expressam a dependência sintática de um tema relativo com respeito ao seu determinante. Prefixos dessa natureza foram identificados por esse autor em línguas Tupí, Karíb e Macro-Jê. A função e distribuição dos prefixos relacionais Tupí serão ilustrados no decorrer deste estudo com dados do Mawé, que é uma das línguas desse agrupamento genético que mantém seu sistema de prefixos relacionais ativos (cf. Fanceschini 1999, 2001, 2002), como são também os casos de várias línguas conservadoras da família Tupí-Guaraní (cf. Cabral 2001), da língua Mundurukú (família Mundurukú) (cf. Gomes 2006) e de línguas da família Tuparí, como o Makuráp (Braga 2005, 2007) e o Tuparí (Alves 2002, 2004).

$\mathrm{O}$ artigo encontra-se organizado da forma seguinte. Na sessão 2 tecemos algumas considerações sobre a noção de prefixos relacionais desenvolvida por Rodrigues (1952, 1953, 1981[2010]), para, em seguida, apresentar, na seção 3, uma descrição sincrônica dos prefixos relacionais do Mawé, com base em Franceschini (1999). Nessa mesma sessão apresentamos as marcas de pessoa do Mawé, descritas por Franceschini (op. cit.), pela fundamental importância que essas marcas tiveram nas mudanças aqui focalizadas. Na sessão 4 desenvolvemos uma hipótese reconstrutiva da história dos relacionais em Mawé, buscando explicar as respectivas formas fonológicas atuais desses prefixos, a sua distribuição em associação com marcas pessoais e a passagem de temas de uma classe a outra ou a novas subclasses temáticas. Na seção 5 apresentamos algumas considerações gerais sobre a hipótese demonstrada.

O presente estudo se apoia em dados do Mawé coletados e analisados por Franceschini ${ }^{5}$ e na reconstrução fonológica e lexical do Proto-Tupí de autoria de Rodrigues $(2005,2010)$ e de Rodrigues e Dietrich (1997), e nas reconstruções gramaticais desenvolvidas por Cabral (2007a, 2007b), por Rodrigues, Cabral e Corrêa da Silva (2006), por Cabral e Franceschini (2011), por Corrêa da Silva (2011), e por Rodrigues e Cabral (2012).

\section{Considerações sobre a noção de prefixos relacionais}

Prefixos relacionais foram descritos por Rodrigues (1952, 1953, 1981[2010]) como prefixos integrantes de um paradigma flexional cuja função é a de marcar em temas relativos a contiguidade sintática (+/-contíguo) dos seus respectivos determinantes, as relações de dependência que mantêm entre si (cf. Cabral 2001), além de sublinhar se o determinante é genérico ou não.

5 Os dados do Mawé que fundamentam este estudo pertencem ao acervo linguístico Mawé organizado por Dulce do Carmo Franceschini, o qual inclui dados por ela coletados junto aos Mawé ao longo dos últimos 20 anos, assim como registros em áudio e em vídeo de reuniões de professores e membros das comunidades Mawé, além de outras situações comunicativas entre falantes do Mawé. 
Ressaltamos que, já em seu estudo A Case of Affinity among Tupí-Karíb and Macro-fê (1989 [2009]), Rodrigues havia proposto um conjunto de prefixos relacionais para o que teria sido o ancestral desse agrupamento genético. Nesse estudo, Rodrigues (p. 159) destacou:

Although there is not yet enough lexical comparative data for establishing regular phonological correspondences among MacroJê on the one hand and Tupí and Karíb, on the other (for the latter two with one another a first list of regular correspondences was contributed in Rodrigues 1985) as well as among the branches of Macro Jê (for Jê-Karajá and Jê-Maxakalí see Davis 1968, for JêOfayé see Gudschinsk 1971), the most probable ancestor of the morphosyntactic pattern examined here could not be too different from the following (where D stands for a probable coronal stop).

O conjunto de prefixos relacionais proposto por Rodrigues é o seguinte:

\begin{tabular}{|l|l|l|}
\hline & Classe A & Classe B \\
\hline & Temas iniciados V & Temas iniciados C \\
\hline Contíguo & ${ }^{*} D-$ & ${ }^{*} \varnothing-$ \\
\hline Não contíguo & ${ }^{*} t s^{-}$ & ${ }^{*}{ }^{-}$ \\
\hline
\end{tabular}

Como ressaltado por Rodrigues (op. cit.), tanto a forma fonológica do prefixo marcando a contiguidade do determinante, quanto a forma do prefixo marcando a não contiguidade do determinante da Classe temática $\mathrm{A}$ teriam sido alveolares, o contíguo oclusivo e o não contíguo africado.

Com base nas reconstruções fonológicas propostas para o Proto-Tupí por Rodrigues (2005, 2007, 1981[2010]), Cabral (2007a, 2007b) propôs a reconstrução da forma ${ }^{* *} t^{3}$ - para o prefixo relacional de contiguidade da classe II do ProtoTupí. Rodrigues e Cabral (2012) incorporaram essa forma na reconstrução do prefixo relacional não contíguo do Proto-Tupí.

\begin{tabular}{|l|l|l|}
\hline \multicolumn{2}{|l|}{ RELACIONAIS DO PROTO TUPÍ } \\
\hline & Classe I & Classe 2 \\
\hline Contíguo $\left(\mathrm{R}^{1}-\right)$ & ${ }^{* *} t^{\gamma_{-}}$ & ${ }^{* *} \varnothing-$ \\
\hline Não contíguo $\left(\mathrm{R}^{2}-\right)$ & ${ }^{*}{ }^{*} i_{-}$ & ${ }^{* *} C_{-}$ \\
\hline Genérico $\left(\mathrm{R}^{4}-\right)$ & ${ }^{* *} m-\infty{ }^{* *} \varnothing-$ & ${ }^{* *} \varnothing-\left(\infty \sim{ }^{* *} t-\right)$ \\
\hline
\end{tabular}

Rodrigues e Cabral (2012) consideram a forma ${ }^{* *} t^{3}$ - para o prefixo $R^{1}$ - da Classe temática II, fundamentados nos reflexos do Proto-Tupí ${ }^{* *} t^{?}$, presentes em dados de línguas representativas de todas as famílias do tronco Tupí. De acordo com Rodrigues (2007), Proto-Tupí ${ }^{* *} t^{t}$ tem os seguintes reflexos nas famílias desse tronco: 
Quadro 1. Alguns reflexos do Proto-Tupí * * $t^{?}$

\begin{tabular}{|c|c|c|c|c|c|}
\hline $\mathrm{PT}$ & ${ }^{* *} t^{?}$ & $\begin{array}{l}{ }^{*}{ }^{*} t^{2} a(j) t^{2} u \\
\text { 'tatu' }\end{array}$ & $\begin{array}{l}{ }^{* *} \boldsymbol{t}^{?} \boldsymbol{i} \boldsymbol{\eta} \\
\text { 'fumaça, branco' }\end{array}$ & $\begin{array}{l}{ }^{* *} \boldsymbol{t}^{?} \mathbf{i} \\
\text { 'urina' }\end{array}$ & $\begin{array}{l}{ }^{*} \boldsymbol{p}_{\boldsymbol{i}} \boldsymbol{t}^{?} \boldsymbol{u} \\
\text { 'respirar' }\end{array}$ \\
\hline PTG & ${ }^{*} t$ & ${ }^{*}$ tatu & ${ }^{*} t i \eta$ & ${ }^{*} t \dot{t}$ & \\
\hline AW & $t$ & tatu & tin & $t \dot{t}$ & pitu \\
\hline MA & $s^{-},-h_{-}^{-}$ & sahu & hin & $s \dot{t}$ & pihu \\
\hline $\begin{array}{l}\mathrm{MU} \\
\mathrm{KU}\end{array}$ & $d$ & $\begin{array}{l}\text { daidu } \\
\text { lajləð }\end{array}$ & din & $-\partial i$ & pido \\
\hline $\mathrm{JU}$ & & & $s \tilde{l}-a$ & & \\
\hline $\mathrm{AR}$ & $t s$ & tsosji; & nin-0 & & \\
\hline $\mathrm{KA}$ & $s$ & sosit, & nin-a & si & \\
\hline $\mathrm{TU}$ & $\begin{array}{l}s /{ }_{-}, \# h- \\
I_{-} V, \varnothing / V_{-} V\end{array}$ & & sin, & sio & \\
\hline MK & & tajto & & & pito \\
\hline $\mathrm{ME}$ & & tato & & $t i$ & \\
\hline $\mathrm{PU}$ & $j$ & jajo. & & & \\
\hline $\mathrm{CL}$ & $s$ & & $w a-n \tilde{\imath} \eta$ & & \\
\hline PA & $l$ & & nín, & & \\
\hline GA & & & $d \tilde{\imath} k$ & & \\
\hline RA & $j$ & jájo & & & \\
\hline
\end{tabular}

Como veremos adiante, o prefixo relacional de não contiguidade em Mawé, tem as formas $s$ - ou $h$-. O prefixo $R^{1}$ - sinaliza em um tema determinado que este forma uma unidade sintática com o seu determinante, que é a expressão imediatamente precedente. O prefixo relacional $R^{2}$-sinaliza que o determinante de um tema não forma com este uma unidade sintática e que é diferente do falante e do ouvinte, e o prefixo relacional $R^{4}$ - sinaliza em um tema que o seu determinante é genérico e humano. A distribuição de temas nominais Tupí com os alomorfes do prefixo $R^{1}$ - é a base para postular a divisão formal de duas classes temáticas nominais para o Proto-Tupí: Classe I e Classe II. As duas classes são ainda divididas em duas subclasses de acordo com a combinação de seus respectivos temas com os alomorfes dos relacionais de não continuidade $R^{2}$ - e $R^{4}$ - (cf. Rodrigues 1981 [2010:17]), em que $R^{1}$-, $R^{2}$-, e $R^{4}$ - foram rotulados especificamente para o Tupinambá como 14, 15, e 18, respectivamente). 
Reconhecemos que há línguas Tupí que apresentam desvios dos padrões de distribuição dos prefixos relacionais originais. São desvios resultantes de mudanças de naturezas distintas (fonológicas, morfossintáticas, semânticas, dentre outras), as quais teriam motivado o movimento de temas de uma classe a outra e a criação de novas classes temáticas ou subclasses. Reconhecemos também que há línguas que perderam os reflexos dos prefixos relacionais do Proto-Tupí, total ou parcialmente. Mas as descrições linguísticas de várias línguas de diferentes famílias Tupí evidenciam que os seus respectivos prefixos relacionais continuam plenamente ativos, como é o caso do Mawé.

\section{Prefixos relacionais do Mawé em nomes e temas estativos, uma abordagem sincrônica}

Em Mawé, núcleos de construções genitivas e de predicados estativos têm em comum o fato de serem flexionados por prefixos que marcam a contiguidade sintática do determinante - um nome ou um prefixo pessoal. Reproduzimos, em seguida, os prefixos relacionais e os prefixos pessoais descritos para o Mawé por Franceschini (1999), visto que a combinação destes últimos com temas flexionados pelos primeiros é primordial para a hipótese reconstrutiva ora apresentada.

Segundo Franceschini (1999), os nomes do Mawé podem ser divididos em alienáveis e inalienáveis, estes últimos agrupados em classes e subclasses, segundo sua combinação com os prefixos relacionais (Franceschini 1999:26).

Tabela 1. Prefixos relacionais agrupados de acordo com classes temáticas e subclasses, adaptado de Franceschini (1999:26)

\begin{tabular}{|l|l|c|c|c|}
\hline \multirow{2}{*}{$\begin{array}{l}\text { Classes } \\
\text { temáticas }\end{array}$} & $\begin{array}{l}\text { Subclasses } \\
\text { temáticas }\end{array}$ & \multicolumn{3}{|l|}{ Prefixes relacionais } \\
\hline & & Contíguo & Não contíguo & Genérico \\
\hline \multirow{2}{*}{$\begin{array}{l}\text { Classe I - } \\
\text { Inalienáveis }\end{array}$} & a) & i- $\sim \varnothing$ & i- & $\varnothing-$ \\
\hline & b) & i- $\sim \varnothing$ & i- & N- \\
\hline $\begin{array}{l}\text { Classe II - } \\
\text { Inalienáveis }\end{array}$ & a) & h- $\sim \mathrm{s}^{-}$ & h- & s- \\
\cline { 2 - 5 } & b) & h- $\sim$ j- & h- & j- \\
\cline { 2 - 5 } & c) & h- $\sim \varnothing$ & h- & s- \\
\hline & $\begin{array}{l}\text { d) e- 'mediador } \\
\text { de posse' }\end{array}$ & h- $\sim \varnothing-$ & h- & -- \\
\hline
\end{tabular}

Os seguintes exemplos ilustram os prefixos relacionais do Mawé combinados com temas em relações de determinação com os seus respectivos determinantes ${ }^{6}$ :

6 Abreviaturas: 1 = primeira pessoa; 2 = segunda pessoa do singular; 13 = primeira pessoa exclusiva; 23 = segunda pessoa do plural; 3 = terceira pessoa do singular; АCтT = Actante; 


\section{Classe Ia}

\section{Contíguo}

mana Ø-?asap i-kahu

mana R-cabelo R-ser.bonito

'o cabelo da mana é bonito'

\section{Não contíguo}

i-?asap i-kahu

R-cabelo R-ser.bonito

'o cabelo dele(a) é bonito'

\section{Genérico}

$\varnothing$-?asap mi-?u pe

G-cabelo nom-comer LOC

'tem cabelo na comida'

\section{Classe Ib}

\section{Contíguo}

u-i-ty

1-R-mãe

Paulo Ø-ty

Paulo R-mãe

'mãe de Paulo'

\section{Genérico}

ny

G.mãe

\section{Classe IIa}

\section{Contíguo}

$\begin{array}{llc}\text { aware } & \text { S-uwaipo } & \text { i-?iwo:p } \\ \text { cachorro } & \text { R-rabo } & \text { R-ser.longo } \\ \text { 'o rabo do cachorro é comprido' }\end{array}$

$\mathrm{AR}=$ Arikém $; \mathrm{AW}=$ Awetí CAUS $=$ causativo $; \mathrm{CL}=$ Cinta-Larga CORR. $=$ correferencial; EXCL. = exclusiva; $\mathrm{G}$ = genérico; $\mathrm{GA}$ = Gavião; $\mathrm{KA}=$ Karitiána; $\mathrm{KU}=$ Kuruáya; $\mathrm{MA}=$ Maué; $\mathrm{ME}=$ Mekéns; $\mathrm{MK}=$ Makurap; M.P. $=$ mediador de posse; $\mathrm{MU}=$ Mundurukú; $\mathrm{PA}=$ Paitér; $\mathrm{PL}$ = plural; POSP.$=$ posposição $;$ PT $=$ Proto-Tupí; PTG = Proto-Tupí-Guaraní; PU = Puruborá; $\mathrm{R}=$ relacional; $\mathrm{RA}=$ Ramaráma . 


\section{Não contíguo}

h-uwaipo i-?iwo:p

$\mathrm{R}$-rabo R-ser longo

'(o) rabo dele(dela) é comprido'

\section{Genérico}

mi?i s-uwaipo

DEIT. G.rabo

'isso é rabo'

\section{Classe IIb}

\section{Contíguo}

kurum j-iin h-ati

menino R-dente $\mathrm{R}$-doer

'o dente do menino está doendo'

\section{Não contíguo}

h-iin h-ati

$\mathrm{R}$-dente $\mathrm{R}$-doer

'o dente (dele(a)) está doendo'

\section{Genérico}

ho?o-kup j-iin ko?i jup pe

PL-existir G-dent PL DEIT POSP.

'tem muitos dentes aqui'

\section{Classe IIc}

\section{Contíguo}

u-h-ewa

1-R-face

'minha face'

uru-Ø-ewa

13-R-face

'nossa (excl.) face'

\section{Não contíguo}

h-ewa

$\mathrm{R}$-face

'face dele(a)' 


\section{Genérico}

s-ewa

$\mathrm{R}$-face

'face (genérico)'

\section{Contíguo}

ariukere $\varnothing$-eha i-wato

bicho-preguiça R-olho R-ser.grande

'o olho do bicho-preguiça é grande'

\section{Não contíguo}

h-eha i-wato

R-olho R-ser grande'

'o olho (dele(a)) é grande'

\section{Genérico}

holo-kup s-eha ko?i wa?ã pe PL-existir G-olho PL panela POSP.

'tem muitos olhos na panela'

\section{Classe IId}

\section{Contíguo}

kurum Ø-e-?iara i-pakup

menino R-M.P.-canoa R-ser novo'

'a canoa do menino é nova'

\section{Não contíguo}

h-e-Piara i-pakup

R-M.P.-canoa R-ser.novo

'a canoa (dele(a)) é nova.'

\section{Genérico}

?iara i-pakup

canoa R-ser.novo

'(a) canoa é nova) 


\section{Contíguo}

u-h-e-sokpé

1-R-M.P.-roupa

'minha roupa'

\section{Genérico}

a-ti-mo-tin

1-ACTT-CAUS-manchar

'eu pintei a roupa'

\section{Não contíguo}

Ø-e-sokpé

R-M.P.-roupa

'roupa dele(a)'

Quanto aos prefixos pessoais, estes se distribuem em três séries, distinguindo verbos de processo (ativos e médios) de verbos de estado e de núcleos de predicados possessivos. Uma série codifica o possuidor e também o actante único de predicados estativos, outra codifica o primeiro actante dos verbos ativos e o actante único dos verbos médios, outra codifica, ao mesmo tempo, o agente e o paciente de verbos ativos, mas é restrita a relações em que uma primeira pessoa age sobre uma segunda pessoa.

Table 2. Prefixos pessoais do Mawé adaptados de Franceschini (1999:98;116)

\begin{tabular}{|c|c|c|c|}
\hline Glosa & $\begin{array}{c}\text { Prefixos } \\
\text { possessivos/ } \\
\text { inativos (p.98) }\end{array}$ & $\begin{array}{l}\text { Prefixos } \\
\text { agentivos } \\
\text { (p.116) }\end{array}$ & $\begin{array}{c}\text { Prefixos sagitais ou } \\
\text { portemanteaux } \\
\text { (p.116) }\end{array}$ \\
\hline 1 & $\mathrm{u}-$ & a- & \\
\hline 1 incl. & $a^{-}$ & wa- & \\
\hline 1 excl. & uru- & uru- & \\
\hline 2 & e- & e- & \\
\hline $2 \mathrm{pl}$. & e- & ewe- ewei- & \\
\hline 3não-corr. & $\mathrm{i}-/ \varnothing-$ & $\varnothing-$ & \\
\hline 3não-corr. pl & i?atu- & ta?ato- $\sim$ te?ero- & \\
\hline 3 corr. & to- & to- & \\
\hline 3 corr.pl & ta?ato- & talato- te?ero- & \\
\hline $1 \rightarrow 2$ & & & moro- $\sim$ woro- $\sim$ oro- \\
\hline $1 \rightarrow 23$ & & & $\begin{array}{l}\text { moro-holo- woro-holo- } \\
\sim \text { oro-ho?o- }\end{array}$ \\
\hline
\end{tabular}

Os seguintes exemplos ilustram a combinação de prefixos relacionais com pronomes pessoais possessivos (inativos) determinando temas das classes I e IIa, b e c. 


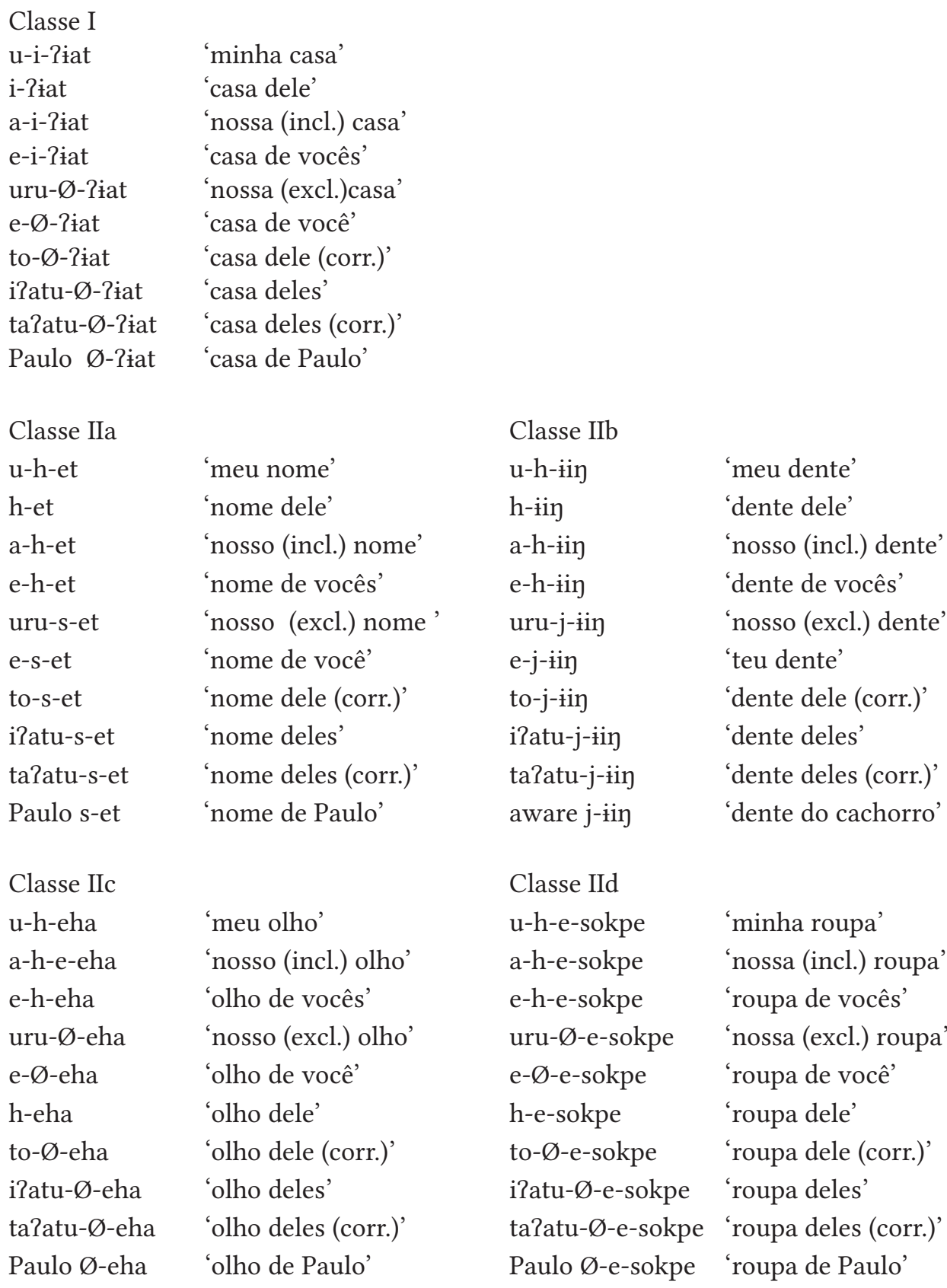

O mais interessante a respeito da distribuição dos alomorfes desses prefixos no Mawé é que, como bem descreveu Franceschini (1999), essa distribuição é condiciona por prefixos pessoais. Como pode ser observado na tabela 2, os prefixos de primeira pessoa do singular, de primeira pessoa do plural inclusiva, de segunda pessoa do plural condicionam as formas $i$ - e $h$-do prefixo relacional de contiguidade, respectivamente em temas das classes I e 
II, enquanto que as demais pessoas, ou seja a segunda do singular, a primeira exclusiva, a terceira pessoa do plural não correferencial e as terceiras pessoas correferenciais (singular e plural) condicionam as formas $\varnothing$ - e $-s^{-} \sim j-\sim \varnothing$ - do mesmo prefixo, respectivamente em temas das classes I e II.

\section{Problemas na correlação dos relacionais do Mawé com os relacionais de outras línguas Tupí-Guaraní}

A alomorfia dos prefixos relacionais do Mawé representa um quebra-cabeça quando se busca correlacionar as suas formas com as de outras línguas Tupí, como por exemplo, com as formas de línguas da família Tupí-Guaraní, com as quais o Mawé compartilha uma história comum (cf. Rodrigues e Dietrich 1997; Corrêa-da-Silva 2011; Rodrigues e Cabral 2012).

O Mawé, assim como as línguas Tupí-Guaraní, distingue duas classes principais de temas, a dos relativos (ou inalienáveis) e a dos absolutos (ou alienáveis). Nas línguas Tupí-Guaraní, assim como em outras famílias do tronco Tupí, os temas relativos se dividem em duas classes principais, Classes I e II, de acordo com a sua distribuição com os alomorfes do prefixo relacional de contiguidade, um vazio de conteúdo fonológico, representado por $\varnothing$-, e outro cuja forma fonológica corresponde a uma consonante coronal, mais ou menos anterior, dependendo da família. O que diferencia o Mawé das línguas de outras famílias é o fato de que o alomorfe do prefixo relacional de contiguidade da classe I, que em Tupí-Guaraní, por exemplo, é vazio de forma fonológica, em Mawé tem, além deste, outro de conteúdo fonológico $i$ -

\begin{tabular}{|l|l|l|l|}
\hline \multicolumn{2}{|l|}{ Mawé } & \multicolumn{2}{l|}{ Asuriní do Tocantins } \\
\hline Classe Ib & \multicolumn{2}{l|}{ Classe Ia } \\
\hline u-i-ti & 'minha mãe' & se Ø-hí-a & 'minha mãe' \\
\hline uru-Ø-ti & 'nossa (excl.) mãe' & ore Ø-hi-a & 'nossa (excl.) mãe' \\
\hline
\end{tabular}

Da mesma forma, o alomorfe do prefixo relacional de contiguidade da classe II, que nas outras línguas Tupí tem um só alomorfe foneticamente coronal, no Mawé possui quatro alomorfes, dois coronais $s-, j$-, um sem forma fonológica $\varnothing$ - e um glotálico $h$ -

\begin{tabular}{|l|l|l|l|}
\hline \multicolumn{2}{|l|}{ Mawé } & \multicolumn{2}{l|}{ Asuriní do Tocantins } \\
\hline Classe IIa & \multicolumn{2}{l|}{ Classe IIa } \\
\hline u-h-et & 'meu nome' & se r-er-a & 'meu nome' \\
\hline uru-s-et & 'nosso (excl.) nome' & ore r-er-a & 'nosso (excl.) nome' \\
\hline
\end{tabular}




\begin{tabular}{|c|c|c|c|}
\hline \multicolumn{2}{|l|}{ Mawé } & \multicolumn{2}{|c|}{ Asuriní do Tocantins } \\
\hline Classe IIb & & Classe IId & \\
\hline $\mathrm{u}-\mathrm{h}$-iin & 'meu dente' & se $\mathrm{r}-\mathrm{is}-\mathrm{a}$ & 'meu dente' \\
\hline uru-j-iin & 'nosso (excl.) dente' & ore $\mathrm{r}$-is-a & 'nosso (excl.) dente' \\
\hline
\end{tabular}

\begin{tabular}{|c|c|c|c|}
\hline \multicolumn{2}{|l|}{ Mawé } & \multicolumn{2}{|c|}{ Asuriní do Tocantins } \\
\hline Classe IIc & & Classe IIc & \\
\hline u-h-eha & 'meu olho' & se r-eha & 'meu olho' \\
\hline uru-Ø-eha & 'nosso (excl.) olho' & ore r-eha & 'nosso (excl.) olho' \\
\hline
\end{tabular}

\section{Uma proposta de reconstrução interna dos relacionais do Proto-Mawé}

Franceschini (1999:63) descreve além da série de prefixos pessoais, uma série de pronomes independentes para o Mawé, a qual reproduzimos abaixo:

\begin{tabular}{|ll|}
\hline Glosa & Pronomes pessoais \\
\hline 1 & uito \\
\hline 2 & en \\
\hline 1 incl. & aito \\
\hline 1 excl. & uruto \\
\hline $2 \mathrm{pl}$ & eipe \\
\hline 3 & mi?i \\
\hline
\end{tabular}

As formas para 1, 1 incl., e 1 excl. têm em comum a sequência de segmentos to, provavelmente vestígios presentemente inanalisáveis de uma antigo morfema da língua. Isolada a sequência to dessas formas pronominais, restam as formas $u j$-, aj- e $u r u$-, respectivamente para 1, 1 incl. e 1 excl. Combina fonologicamente com as formas $u j-$, aj- a forma ej-, de ejpe, abstraída a sequência pe. Propomos aqui, seguindo Cabral (2007b) e Cabral, Rodrigues e Franceschini (2011), que as formas para a primeira pessoa do singular, para a primeira inclusiva e para a segunda do plural teriam sido em estágio anterior da história da língua Mawé, $u j-, a j-$ e $e j-$, como mostrado no quadro seguinte:

\begin{tabular}{|l|l|}
\hline Glosa & Prefixos possessivos/inativos (p.98) \\
\hline 1 & uj- \\
\hline 1 incl. & aj- \\
\hline 1 excl. & uru- \\
\hline 2 & e- \\
\hline 2 pl & ej- \\
\hline 3não-corr. & i-/Ø- \\
\hline 3não-corr.pl & i?atu- \\
\hline
\end{tabular}


De mãos dessa hipótese, podemos então explicar a alomorfia existente no sistema de prefixos relacionais da língua Mawé como resultado de processos fonológicos ocorridos em fronteira de morfema envolvendo prefixos pessoais e prefixos relacionais, em um estágio anterior da língua.

\section{Reconstruindo a história}

Rodrigues (1997) reconstrói formas fonológicas para os dois alomorfes do prefixo relacional de não contiguidade do Proto-Tupí, ${ }^{* *} i$ - e ${ }^{* *} c$-, o primeiro combinando-se com elementos da classe temática I e o segundo com temas da classe temática II. Quanto às formas dos relacionais de contiguidade, Rodrigues (1992) postula que o que se combina com a classe temática I teria sido vazio de forma fonológica, representando-o por ${ }^{* *} \varnothing$-, deixando a forma do relacional que se combinaria com temas da classe II em aberto. Cabral (2007), com base na reconstrução fonológica do Proto-Tupí proposta por Rodrigues (2007) e considerando a forma dos prefixos relacionais através das diferentes famílias Tupí, propõe a reconstrução do alomorfe ${ }^{* *} t^{3}$ - para o prefixo relacional que teria marcado a contiguidade do determinante de temas da classe II. Considerando essas reconstruções, o quadro de relacionais do PT teria tido a seguinte configuração:

\begin{tabular}{|l|c|c|}
\hline \multirow{4}{*}{} & \multicolumn{2}{|c|}{ Prefixos Relacionais do PT } \\
\cline { 2 - 3 } & $\begin{array}{c}\text { Prefixo relacional de } \\
\text { contiguidade }\end{array}$ & $\begin{array}{c}\text { Prefixo relacional de } \\
\text { não contiguidade }\end{array}$ \\
\cline { 2 - 3 } Classe I & $\varnothing-$ & i- \\
\cline { 2 - 3 } Classe II & $\mathrm{t}^{3}-$ & C- \\
\hline
\end{tabular}

Vejamos então os processos fonológicos que teriam sido ativados em fronteira de morfemas envolvendo prefixos pessoais e prefixos relacionais em um estágio anterior do Mawé.

Temas da classe I teriam se combinado originalmente com o alomorfe $\varnothing$ do prefixo relacional de contiguidade, e com o alomorfe $i$ - do prefixo relacional de não contiguidade. Em um momento posterior, a fase off-glide dos prefixos pessoais combinados com temas da classe I teria sido reanalisada como sendo um alomorfe do prefixo relacional de contiguidade, por analogia ao alomorfe da classe II. Entretanto, em combinação com as demais pessoas que não tinham fase off-glide teria continuado $\varnothing$-. 


\section{Classe I}

\begin{tabular}{|c|c|c|c|}
\hline *ui-Ø-ti & $>$ & $\mathrm{u}-\mathrm{i}-\mathrm{t} \dot{\mathrm{i}}$ & 'minha mãe' \\
\hline *ai-Ø-ti & $>$ & $\mathrm{a}-\mathrm{i}-\mathrm{ti}$ & 'nossa (Incl.) mãe' \\
\hline *ei-Ø-ti & $>$ & $e-i-t i$ & 'mãe de vocês' \\
\hline *uru-Ø-ti & $>$ & uru-Ø-ti & 'nossa (Excl.) mãe' \\
\hline *e-Ø-ti & $>$ & e- $\varnothing-\mathrm{ti}$ & 'mãe de você' \\
\hline *to- $\varnothing-\mathrm{t} \dot{\mathrm{x}}$ & $>$ & to- $\varnothing-\mathrm{t} \dot{\mathrm{t}}$ & 'mãe dele (corr.)' \\
\hline *i?atu-Ø-ti & $>$ & i?atu-Ø-ti & 'mãe deles' \\
\hline *ta?atu-Ø-ti & $>$ & ta?atu- $\varnothing-\mathrm{ti}$ & 'mãe deles (corr).' \\
\hline *Paulo Ø-tí & $>$ & Paulo $\varnothing$-tí & 'mãe de Paulo' \\
\hline${ }^{*} \mathrm{i}-\mathrm{ti}$ & $>$ & $\mathrm{i}-\mathrm{ti}$ & 'mãe dele(a)' \\
\hline
\end{tabular}

Quanto aos temas da classe II, postulamos que o segmento fonológico correspondente ao alomorfe do prefixo de contiguidade desta classe teria mudado de ${ }^{* *} t^{3}$ - para $t \rho^{\beta}$ - quando precedido por prefixos pessoais com fase offglide, $\left({ }^{* *} t^{3}->t \rho^{p}-/ \mathrm{i}+\ldots\right)$, fundindo-se com os reflexos do PT ${ }^{*} t f^{\text {, }}$, que mudaram posteriormente para $h$ : ** pičr $\tilde{a}$ 'artelho': TG *pičã : AW pitã: MA pihã: ** eč $a$ 'olho': TG *-eča: Tupinambá - esa: Guaraní A. -eca: AW -eta: MA -eha: JU Jurúna - elá: MU Mundurukú, Kuruáya eta: AR Karitiána aso 'rosto' 'face’: MO Gavião ǐja-kap: RA Káro ča.

Já nas demais situações, a forma do mesmo prefixo relacional teria mudado para $t^{s}:{ }^{*} t^{2} \dot{i}$ 'urina': TG ${ }^{*} t \dot{t}$; AW $t \dot{t} ;$ MA $t s \dot{t}>s \dot{i}$; MU Kuruáya -ði; AR Arikém, Karitiána si; TU Mekéns $t i$.

\section{Classe IIa}

\begin{tabular}{|c|c|c|c|}
\hline *ui-t ${ }^{3}$-et & $>{ }^{*} u-t \int^{3}-e t$ & $>$ u-h-et & 'meu nome' \\
\hline${ }^{*} a i-t^{3}-$ et & $>{ }^{*} \mathrm{a}-\mathrm{t} \int^{3}$-et & $>$ a-h-et & 'nosso (Incl.) nome' \\
\hline *ei-t $t^{3}$-et & $>{ }^{*} \mathrm{e}-\mathrm{t} \int^{3}$-et & $>$ e-h-et & 'nome de vocês' \\
\hline${ }^{*}$ uru-t $\mathrm{t}^{2}$-et & $>{ }^{*}$ uru-ts-et & $>$ uru-s-et & 'nosso(Excl.) nome' \\
\hline$* e-t^{3}-e t$ & $>{ }^{*}$ e-ts-et & $>$ e-s-et & 'nome de você' \\
\hline${ }^{*}$ to- $\mathrm{t}^{3}$-et & $>{ }^{*}$ to-ts-et & $>$ to-s-et & 'nome dele (cor.)' \\
\hline${ }^{*}$ i?atu-t? & $>{ }^{*}$ i?atu ts-et & $>$ i?atu s-et & 'nome deles' \\
\hline *ta?atu-t ${ }^{2}$-et & $>{ }^{*}$ ta?atu ts-et & $>$ ta?atu s-et & 'nome deles (cor.)' \\
\hline${ }^{*}$ kurum $t^{3}$-et & $>{ }^{*}$ kurum ts-et & $>$ kurum s-et & 'nome do menino' \\
\hline
\end{tabular}


Quando o prefixo relacional precedia tema nasal, teria sofrido nasalização, realizando-se com a forma $n$, isso se não fosse precedido por prefixos com fase off-glide, uma vez que neste ambiente a palatalização se aplicaria primeiro, bloqueando a nasalização, como mostra o paradigma do tema para 'dente'.

\begin{tabular}{|c|c|c|c|c|c|}
\hline \multicolumn{6}{|l|}{ Classe IIb } \\
\hline *ui-t t $^{3}$ iin & $>$ & ${ }^{*} \mathrm{u}-\mathrm{t} \int^{3}$-iin & $>$ & u-h-iin & 'meu dente' \\
\hline *ai-t ${ }^{2}-$ iin & $>$ & *a-t $\int^{3}$-iin & $>$ & a-h-iin & 'nosso (Incl.) dente' \\
\hline *ei-t $t^{2}-\mathrm{iin}$ & $>$ & *e-t $\int^{3}$-iin & $>$ & e-h-iin & 'dente de vocês' \\
\hline *uru-t $\mathrm{t}^{3}$-iin & $>$ & *uru-n-iin & $>$ & uru-j-iin & 'nosso (Excl.) dente' \\
\hline$* e-t^{2-\ddot{t i n}}$ & $>$ & *e-n-iin & $>$ & e-n-ïin & 'teu dente' \\
\hline${ }^{*}$ to- $\mathrm{t}^{3}$-iin & $>$ & *to-j-iin & $>$ & to-j-iin & 'dente dele (corr.)' \\
\hline *ilatu-t²-iin & $>$ & *i?atu-n-iin & $>$ & i?atu-n-iin & 'dente deles' \\
\hline${ }^{*}$ ta?atu-t ${ }^{3}-$ iin & $>$ & *ta?atu-n-ïin & $>$ & ta?atu-n-iin & 'dente deles (corr.)' \\
\hline *aware $t^{2}$-iin & $>$ & *aware n-iin & $>$ & aware n-iin & 'dente do cachorro' \\
\hline
\end{tabular}

\begin{tabular}{|c|c|c|c|c|}
\hline \multicolumn{5}{|l|}{ Classe IIc } \\
\hline *ui-t? & $>$ & $\mathrm{u}-\mathrm{t} \int^{3}$-eha & u-h-eha & 'meu olho' \\
\hline${ }^{*}$ ai-t $\mathrm{t}^{2}$ eha & $>$ & $\mathrm{a}-\mathrm{t} \int^{3}$-eha & a-h-eha & 'nosso (Incl.) olho' \\
\hline${ }^{*}$ ei-t ${ }^{2}$-eha & $>$ & e-t $\int^{3}$-eha & e-h-eha & 'olho de vocês' \\
\hline${ }^{*}$ uru- $t^{2}$-eha & $>$ & *uru-ts-eha & uru-Ø-eha & 'nosso (Excl.) olho' \\
\hline${ }^{*} \mathrm{e}-\mathrm{t}^{3}-\mathrm{eha}$ & $>$ & *e-ts-eha & e-Ø-eha & 'olho de você' \\
\hline *to-t $\mathrm{t}^{3}$-eha & $>$ & *to-ts-eha & to-Ø-eha & 'olho dele (cor.)' \\
\hline *i?atu-t ${ }^{3}$-eha & $>$ & *i?atu-ts-eha & i?atu-Ø-eha & 'olho deles' \\
\hline${ }^{*}$ ta?atu-t ${ }^{3}$-eha & $>$ & *ta?atu-ts-eha & ta?atu-Ø-eha & 'olho deles (cor.)' \\
\hline \multirow[t]{2}{*}{${ }^{*}$ kurum $t^{2}$-eha } & $>$ & ${ }^{*}$ kurum ts-eha & kurum Ø-eha & 'olho do menino' \\
\hline & & & s-eha & 'olho dele(a)' \\
\hline
\end{tabular}

\begin{tabular}{|c|c|c|c|}
\hline \multicolumn{4}{|l|}{ Classe IId } \\
\hline *ui-t ${ }^{2}$-e-sokpe & ${ }^{*} \mathrm{u}-\mathrm{t} \int^{3}$-e-sokpe & u-h-e-sokpe & 'minha roupa' \\
\hline${ }^{*}$ ai-t $\mathrm{t}^{2}$-e-sokpe & ${ }^{*} \mathrm{a}-\mathrm{t} \int^{3}$-e-sokpe & a-h-e-sokpe & 'nossa (Incl.) roupa' \\
\hline *ei-t $t^{3}$-e-sokpe & ${ }^{*} \mathrm{e}-\mathrm{t} \int^{2}$-e-sokpe & e-h-e-sokpe & 'roupa de vocês' \\
\hline
\end{tabular}




\begin{tabular}{|c|c|c|c|}
\hline *uru-Ø-e-sokpe & *uru-Ø-e-sokpe & uru-Ø-e-sokpe & 'nossa (Excl.) roupa' \\
\hline *e-Ø-e-sokpe & *e-Ø-e-sokpe & e-Ø-e-sokpe & 'roupa de você' \\
\hline *to-Ø-e-sokpe & *to-Ø-e-sokpe & to- $\varnothing$-e-sokpe & 'roupa dele (cor.)' \\
\hline *i?atu-Ø-e-sokpe & *i?atu-Ø-e-sokpe & i?atu-Ø-e-sokpe & 'roupa deles' \\
\hline *ta?atu-Ø-e-sokpe & *ta?atu-Ø-e-sokpe & ta?atu-Ø-e-sokpe & 'roupa deles (cor.)' \\
\hline *kurum Ø-e-sokpe & *kurum Ø-e-sokpe & kurum Ø-e-sokpe & 'roupa do menino' \\
\hline sokpe & sokpe & sokpe & 'roupa' \\
\hline
\end{tabular}

Note-se que o morfema $e$-constitui sozinho a Classe IId. Este morfema continua plenamente ativo, como mostra o exemplo seguinte no qual $-e$ se combina com um empréstimo do Português:

mio e-i-pusu ti ra'yn e-h-e-documento dem.v poss.-r-língua part. asp. 2-r-poss-documento 'é vossa língua que é vosso documento'

Como o relacional de continuidade $i$-, que foi uma inovação, tinha a mesma forma fonológica do antigo relacional de não contiguidade $i$ - dos temas da classe I, o relacional de não contiguidade da classe II passou a ter a mesma forma fonológica do prefixo relacional de contiguidade desenvolvido no contexto de marcas pessoais com off-glide, como mostram os exemplos seguintes.

\begin{tabular}{|c|l|l|l|l|}
\hline u-i-ti & 'minha mãe' & $=$ & u-h-et & 'meu nome' \\
\hline e-i-tí & 'mãe de vocês' & $=$ & e-h-et & 'nome de vocês' \\
\hline a-i-tí & 'nossa (Incl.) mãe' & $=$ & a-h-et & 'nosso (Incl.) nome' \\
\hline i-tí & $\begin{array}{l}\text { 'mãe dele/dela/ } \\
\text { de alguém/de algum' }\end{array}$ & $=$ & h-et & $\begin{array}{l}\text { 'nome dele/dela/ } \\
\text { de alguém/de algum' }\end{array}$ \\
\hline
\end{tabular}

Isso explica o fato de que vários temas da Classe II têm $h$ - para contíguo e outro prefixo $h$ - para não contíguo. É importante notar que essa mudança analógica serviu de motivação para que a classe II desenvolvesse um relacional genérico $s$ - com forma similar à forma do relacional de não contiguidade dos temas dessa classe combinados com marcas pessoais sem off-glide:

\begin{tabular}{|l|l|l|l|l|}
\hline uru-Ø-memyt & 'nosso excl. filho(a)' & $=$ & uru-s-et & 'meu nome' \\
\hline e-Ø-memyt & 'filho(a) de você' & $=$ & e-s-et & 'nome de você' \\
\hline to-Ø- memyt & 'filho(a) dela (corr.)' & $=$ & to-s-et & 'nome dele(a) (corr.)' \\
\hline
\end{tabular}




\begin{tabular}{|l|l|l|l|l|}
\hline ilatuti-Ø-memyt & $\begin{array}{l}\text { 'filho(a) delas } \\
\text { (não corR.) }\end{array}$ & $=$ & i?atuti-s-et & $\begin{array}{l}\text { 'nome deles(as) } \\
\text { (não coRr.) }\end{array}$ \\
\hline$\varnothing$-memyt & GEN-filho(a) & & s-et & GEN-nome \\
\hline
\end{tabular}

Por outro lado, esperar-se-ia que temas como - eha 'olha' e -ewa 'face' se combinassem com o alomorfe $s^{-}$nas pessoas que tinham off-glide. Mas, contrariamente ao esperado, o alomorfe que ocorre é $\varnothing$-, o que os distingue como pertencentes a uma subclasse distinta.

\section{Considerações finais}

Em suma, a partir dos dados sincrônicos do Mawé e das reconstruções fonológicas e morfológicas feitas para o Proto-Tupí por Rodrigues (2005, 2007), e por Rodrigues e Cabral (2012) foi possível reconstituir estágios anteriores desses prefixos e identificar quais os fatores internos que contribuíram para mudanças por eles sofridas. Este estudo mostrou também que os dados atuais do Mawé fornecem indicações claras de que a função relacional continua necessária ao estabelecimento de relações de subordinação e determinação entre um tema relativo e o seu determinante. O Mawé soma-se, nesse sentido, à família Tupí-Guaraní, ao Mundurukú (fam. Mundurukú), ao Tuparí e ao Makuráp (família Tuparí), nas quais a função relacional manifestada por meio de morfologia prefixal continua ativa.

\section{Referências}

Alves, Polina. 2002. Flexão relacional em Tupari e em Tupí-Guaraní. In Aryon D. Rodrigues, Ana Suelly A. C. Cabral (eds.), Línguas indígenas brasileiras: fonologia, gramática e história. Atas do I Encontro Internacional do grupo de trabalho sobre línguas indígenas da ANPOLL, Belém, 269-273.

Alves, Poliana Maria. 2004. O léxico do Tupari: proposta de um dicionário bilíngue. Tese (Doutorado em Linguística), Universidade Estadual Paulista, Araraquara.

Braga, Alzerinda de Oliveira. 2005. Aspects morphosyntaxiques de la langue Makurap/ Tupi. Tese (Doutorado em Linguística) - Université de Toulouse - Le Mirail.

Braga, Alzerinda de Oliveira. 2007. A construção genitiva em Makurap. In Aryon D. Rodrigues and Ana Suelly A. C. Cabral (eds.), Linguas e Culturas Tupí I, 291-299. Campinas: Curt Nimuendajú; Brasília: LALI/UnB.

Cabral, Ana Suelly A. C. 2001. Prefixos relacionais na família Tupi-Guarani. Boletim da Abralin 25:213-262, Fortaleza.

Cabral, Ana Suelly A. C. 2007a. Prefixos Relacionais em Mawé e em Awetí, uma hipótese reconstrutiva. Trabalho apresentado durante o II Encontro Internacional sobre Línguas e Culturas Tupí, Universidade de Brasília. (ms.) 
Cabral, Ana Suelly A. C. 2007b. Fundamentando a Reconstrução de Prefixos Relacionais para o Proto-Tupí. Caderno de Resumos, pp. 438-39, V Congresso Internacional da ABRALIN, Belo Horizonte, MG.

Cabral, Ana Suelly A. C., Aryon d. Rodrigues e Dulce do Carmo Franceschini. 2011. Reconstrução interna dos prefixos relacionais da língua Mawé. Trabalho apresentado durante o Primeiro Encontro Internacional de Arqueologia e Linguística Histórica das Línguas Indígenas Sul-Americanas, Universidade de Brasília. (ms)

Corrêa da Silva, Beatriz Carretta. 2011. Mawé/Awetí/Tupí-Guaraní: relações linguísticas e implicações históricas. Tese (Doutorado em Linguística), Universidade de Brasília.

Franceschini, Dulce. 2001. As classes de nomes em Sateré-Mawé. Anais do $2^{\circ}$ Congresso Internacional da Abralin. Online: http://sw.npd.ufc.br/abralin/anaiscon2int-sum.html

Franceschini, Dulce. 2002. A voz inversa em Sateré-Mawé. In Aryon D. Rodrigues, Ana Suelly A. C. Cabral (eds.), Línguas indígenas brasileiras: fonologia, gramática e história. Atas do I Encontro Internacional do grupo de trabalho sobre línguas indígenas da ANPOLL, Belém, 222-233.

Franceschini, Dulce. 1999. La langue Sateré Mawé, Description et analyse Morphosyntaxique. Tese (Doutorado em Linguística). Université de Paris VII (Denis Diderot).

Gomes, D. M. Estudo Morfológico e sintático da Língua Mundurukú (Tupí). 2006. Tese (Doutorado em Linguística). Universidade de Brasília.

Rodrigues, Aryon D. 1952. Análise morfológica de um texto Tupi. Logos 15:56-77, Curitiba.

Rodrigues, Aryon D. 1953. Morfologia do verbo Tupi. Letras 1:121-152, Curitiba.

Rodrigues, Aryon D. 1984-1985. Relações internas na família linguística Tupi-Guarani. Revista de Antropologia 27/28:33-53. São Paulo: USP.

Rodrigues, Aryon Dall'Igna. 1985. Evidence for Tupí-Karíb relationships. In: H. E. M. Klein \& L. R. Stark (eds.), South American Indian languages: retrospect and prospect, pp. 371-404. Austin: University of Texas Press.

Rodrigues, Aryon Dall'Igna. 1999. Tupí. In: Dixon, Robert e Aikhenvald, Alexandra (eds.), The Amazonian Languages, pp. 107-124, Cambridge: Cambridge University Press.

Rodrigues, Aryon Dall'Igna. 1989 [2009]. A case of Afinity Among Tupí, Karíb, and Macro-Jê. Revista Brasileira de Línguística Antropológica 1(1):137-177.

Rodrigues, Aryon Dall'Igna. 2009. A Case of Affinity Among Tupí, Karíb, and MacroJê. Revista Brasileira de Linguística Antropológica 1(1):137-161.

Rodrigues, Aryon Dall'Igna. 2005 As vogais orais do Proto-Tupí. In: Aryon Dall'Igna Rodrigues; Ana Suelly Arruda Câmara Cabral. (Org.). Novos estudos sobre línguas indígenas. Brasília: Editora Universidade de Brasília, p. 35-46. 
Rodrigues, Aryon Dall'Igna. 2007. As consoantes do Proto-Tupí. In: Ana Suelly Arruda Câmara Cabral; Aryon Dall'Igna Rodrigues (ed.), Línguas e culturas Tupí, p. 167-203. Campinas: Curt Nimuendaju; Brasília: LALI.

Rodrigues, Aryon Dall'Igna. [1981] 2010. Estrutura do Tupinambá. In Cabral et. al. (org.), Línguas e Culturas Tupí II, Brasília: LALI/UnB; Campinas, SP: Curt Nimuendajú, p. 12-42.

Rodrigues, Aryon D. and Wolf Dietrich. 1997. On the linguistic relationship between Mawe and Tupi-Guarani. Diachronica 14:265-304. Amsterdam.

Rodrigues, Aryon Dall'Igna, Ana Suelly A. C. Cabral e Beatriz C. Correa da Silva. 2006. Evidencias linguísticas para a reconstrução de um nominalizador de objeto ${ }^{* *}$ mi- em Proto-Tupí. Revista de Estudos da Lingua(gem) 4(2):21-39, Vitoria da Conquista, Bahia.

Rodrigues, Aryon D. and Ana Suelly A. C. Cabral. 2012. Tupían. In Lyle Campbell and Verónica Grondona (eds.), The Indigenous Languages of South America 2:495-574. Berlin/Boston: Mouton de Gruyter.

Data recebimento: 04/01/2013

Data aceite: 15/08/2013 